\title{
Primär adaptierende vs. sekundäre Wundverschlüsse bei Infektionen der Hand - Unterschiede und Vorteile
}

\section{Primary Adaptive vs. Secondary Wound Closure in Hand Infections - Differences and Benefits}

Autoren

Institut

\section{Schmauss, T. Finck, J. A. Lohmeyer, M. Reidel, H.-G. Machens, K. Megerle}

Klinik und Poliklinik für Plastische Chirurgie und Handchirurgie, Klinikum rechts der Isar, Technische Universität München, München

\section{Schlüsselwörter \\ - Handinfektion \\ - Wundverschluss \\ - Primärverschluss \\ - Sekundärverschluss}

\section{Key words}

hand infection

- wound closure

- primary closure

- secondary closure

eingereicht $\quad 4.10 .2013$

akzeptiert $\quad 16.12 .2013$

\section{Bibliografie}

DOI http://dx.doi.org/

10.1055/s-0033-1363980

Online-Publikation: 30.1.2014

Handchir Mikrochir Plast Chir

2014; 46: 56-60

(c) Georg Thieme Verlag KG

Stuttgart · New York

ISSN 0722-1819

\section{Korrespondenzadresse}

\section{Dr. Kai Megerle}

Klinik und Poliklinik für Plasti-

sche Chirurgie und Handchi-

rurgie

Klinikum rechts der Isar

Technische Universität

München

München

Kai.Megerle@mri.tum.de

\section{Zusammenfassung}

$\nabla$

Hintergrund: Infektionen der Hand sind ein in chirurgischen Notaufnahmen häufig gesehenes Krankheitsbild. Es wird nach wie vor kontrovers diskutiert, ob nach radikalem Débridement direkt ein primär adaptierender oder in einer zweiten Operation ein sekundärer Wundverschluss durchgeführt werden sollte. Das Ziel dieser retrospektiven Studie war, das Ergebnis nach primär adaptierenden und sekundären Wundverschlüssen nach operativem Débridement bei Handinfektionen zu vergleichen.

Methoden: Alle Patienten, die in unserer Klinik aufgrund von Handinfektionen in den Jahren 2011 und 2012 operiert wurden, wurden retrospektiv nach mindestens 6 Monaten analysiert. Wir schlossen 16 primär adaptierende Wundverschlüsse (PV) und 12 Sekundärverschlüsse (SV) ein. Wir erfassten die Notwendigkeit von ReOperationen, die Dauer des Krankenhausaufenthalts, die Zufriedenheit mit der Behandlung, die Eigenschaften der Narbe und die Beweglichkeit der betroffenen Hand.

Resultate: Kein Patient musste nach einem PV, respektive SV nachoperiert werden. Die durchschnittliche Länge des Krankenhausaufenthalts war in der Gruppe der PV signifikant kürzer als in der Gruppe der SV (3,0 Tage vs. 5,1 Tage; $\mathrm{p}=0,048$ ). Die Zufriedenheit der Patienten mit der Behandlung, der Narbe und der Beweglichkeit der betroffenen Hand waren in beiden Gruppen vergleichbar.

Schlussfolgerung: Diese Studie zeigt, dass Wunden nach einem radikalen Débridement einer Handinfektion ohne Nachteile für den Patienten primär adaptierend verschlossen werden können. Die Länge des Krankenhausaufenthalts ist bei Patienten mit PV signifikant kürzer, was unter dem Aspekt des Patientenkomforts und aus sozio-ökonomischer Sicht wichtig ist. Primär adaptierende und sekundäre Wundverschlüsse zeigten beide gute klinische Ergebnisse.

\section{Abstract \\ $\nabla$}

Background: Hand infections are common surgical emergencies. There are still controversial opinions regarding the ideal timing of wound closure after radical débridement of the infection. The aim of this retrospective study was to compare the outcome of primary adaptive and secondary wound closures after operative débridement in patients with hand infections.

Methods: We retrospectively analysed all infections of the hand treated operatively in our hospital in the years 2011 and 2012 with a follow-up of at least 6 months. We included 16 patients with primary adaptive wound closure (PWC) and 12 patients with secondary wound closure (SWC) in this study. The evaluated parameters were the need for re-operations, the length of hospital stay, the overall satisfaction with the treatment, the characteristics of the scar and the mobility of the hand.

Results: No patient had to be re-operated after PWC or SWC, respectively. Patients in the PWC group were kept significantly shorter as inpatients in comparison to patients in the SWC group (3.0 days vs. 5.1 days; $p=0.048$ ). Overall patient satisfaction with the treatment and the scar was comparable for both groups, as was the re-establishment of the mobility of the treated hand to preoperative levels.

Conclusion: This study shows that wounds after radical débridement for infection of the hand can be closed primarily adaptive without disadvantages for the patient. The length of hospitalisation is significantly shorter if the wound is closed primarily adaptive, a fact that is important for patient comfort and the socio-economic system. Both, primary adaptive and secondary wound closures generally have good outcomes with possible advantages for primary adaptive wound closures concerning the characteristics of the scar. 


\section{Einleitung}

Handinfektionen sind typischerweise bakterielle Infiltrationen der Handregion mit typischen Zeichen einer lokalen Entzündung und stellen ein häufig gesehenes, jedoch oft unterschätztes Krankheitsbild in chirurgischen Notaufnahmen dar [1-5]. Klassischerweise besteht das therapeutische Vorgehen bei einer Handinfektion aus einer adäquaten chirurgische Therapie, einer Immobilisation der Extremität, der Ödemkontrolle und einer antibiotischen Therapie [2,6]. Die Operation beinhaltet ein radikales Débridement des infizierten Gewebes und eine ausgiebige Spülung der Wunde. Traditionell wird die Wunde mit der Idee eines erleichterten Abflusses von eventuell noch infiziertem Wundsekret offen gelassen und erst nach einigen Tagen im Rahmen eines „second looks“ sekundär verschlossen.

Obwohl diese Vorgehensweise aufgrund ihrer niedrigen Komplikationsrate eine hohe Akzeptanz unter Chirurgen genießt, gilt die Notwendigkeit einer zweiten Operation mit dem damit verbundenen stationären Aufenthalt doch als aufwendig und kostenintensiv.

Es wird nach wie vor kontrovers diskutiert, ob ein Primärverschluss der Wunde, also ein einzeitiges Vorgehen, bei Infektionen der Hand oder anderer Körperpartien sinnvoll ist [7]. Das Dogma, dass ein Primärverschluss nach operativer Behandlung eines Abszesses kontraindiziert ist, wurde bereits 1951 von Ellis in Frage gestellt. Der Autor konnte zeigen, dass der Primärverschluss eines anorektalen Abszesses mit einem besseren klinischen Ergebnis als dessen Sekundärverschluss einhergeht [8]. Ein Primärverschluss bei Handinfektionen hätte aufgrund nur einer notwendigen Operation und einer eventuell kürzeren Aufenthaltsdauer im Krankenhaus Vorteile für den Patientenkomfort und wäre auch aus sozio-ökonomischer Sicht erstrebenswert. Dem gegenüber stehen Argumente, dass ein Primärverschluss einer infizierten Wunde verhindert, dass infiziertes Sekret drainieren kann und die Infektion somit nicht abklingen kann. Es gibt überhaupt keine Studien zu Primärverschlüssen bei Infektionen der Hand in der Literatur.

Aufgrund unserer guten Erfahrungen mit adaptierenden Primärverschlüssen nach radikalem Débridement von Infektionen der Hand, haben wir unsere Operationen von Handinfektionen der Jahre 2011 und 2012 nach diesen Gesichtspunkten retrospektiv analysiert.

\section{Methoden}

Im Rahmen dieser retrospektiven Studie analysierten wir alle an unserer Klinik operativ versorgten Handinfektionen im Zeitraum Januar 2011 bis Dezember 2012. Da Handinfektionen oft in der Dienstzeit operiert werden, waren insgesamt 9 Fachärzte für Plastische Chirurgie an den Operationen beteiligt. 2 dieser Operateure präferieren, die Wunde bei Handinfektionen nach dem adäquaten Débridement primär adaptierend zu verschließen, die restlichen 7 Operateure verschließen die Wunde grundsätzlich sekundär. Die Wunden wurden also in Abhängigkeit vom Operateur primär oder sekundär verschlossenen, das Ausmaß der Infektion hatte jedoch keinen Einfluss auf die Strategie. So haben die Operateure, die einen PV präferieren, keinen einzigen Patienten sekundär verschlossen und vice versa. Wir schlossen Patienten mit einem Nachbeobachtungszeitraum von mindestens 6 Monaten, die keine vorangegangene Operationen an der Stelle der Infektion hatten und zu einem Telefoninterview
Tab. 1 Aufteilung des Patientenkollektivs auf Primär- und Sekundärverschluss sowie genauere Spezifizierung beider Gruppen.

\begin{tabular}{|llll|} 
& $\begin{array}{l}\text { Primärver- } \\
\text { schluss }\end{array}$ & $\begin{array}{l}\text { Sekundär- } \\
\text { verschluss }\end{array}$ & p-Wert \\
\hline Anzahl der Patienten & 16 & 12 & \\
\hline männlich & 8 & 7 & 0,72 \\
\hline weiblich & 8 & 5 & 0,72 \\
\hline Durchschnittsalter & 36,5 Jahre & 38,0 Jahre & 0,96 \\
\hline Tierbissverletzungen & 3 & $(21-73)$ & \\
\hline $\begin{array}{l}\text { Menschenbissverlet- } \\
\text { zungen }\end{array}$ & 1 & 5 & 0,23 \\
\hline Keimnachweis & 7 & 1 & 1,00 \\
\hline
\end{tabular}

bereit waren, ein. Nur 36 Patienten erfüllten die ersten beiden Einschlusskriterien, da ein Großteil der Patienten bereits in einem anderen Krankenhaus voroperiert worden war. 8 Patienten konnten trotz mehrfachen Versuchen nicht telefonisch erreicht werden oder erklärten sich nicht bereit, am Telefoninterview teilzunehmen. So konnten wir insgesamt 28 Patienten einschließen, von denen bei 16 ein primär adaptierender Wundverschluss (PV) und bei 12 ein sekundärer Wundverschluss (SV) durchgeführt worden war.

Bei dem Interview fragten wir zum einen, ob eine weitere Operation an der versorgten Hand außerhalb unserer Abteilung notwendig war. Zum anderen wurden die Patienten nach ihrer Operationsnarbe an der Stelle der ausgeheilten Infektion befragt, diese sollte mit „gut“ oder „schlecht“ bewertet werden. Bei einer Bewertung mit „schlecht“ wurde der Grund hierfür erfragt. Weiterhin beinhaltete das Interview die Frage, ob der präoperative Zustand der Beweglichkeit der Hand wiederhergestellt werden konnte oder nicht. Abschließend erfragten wir die Zufriedenheit mit der Behandlung, wobei die Patienten Noten von 1 bis 6 nach dem Schulnotensystem vergeben sollten.

Parallel dazu werteten wir die Akten aller nicht voroperierten Patienten aus und sammelten Informationen über die Anzahl der Operationen, die Anzahl der stationären Nächte, die zugrundeliegende Verletzung und den mikrobiologischen Keimnachweis.

\section{Patientengut}

$\nabla$

Die beiden in dieser Studie verglichenen Patientengruppen waren bezüglich der Geschlechterverteilung, des Alters, der Häufigkeit eines Keimnachweises und der Ursache für die Handinfektion vergleichbar ( $\bullet$ Tab. 1).

Beide Gruppen bestanden etwa zur Hälfte aus Männern und Frauen, das Durchschnittsalter in der PV-Gruppe war 36,5 Jahre, in der SV-Gruppe betrug es 38,0 Jahre. Positive Keimnachweise stellten wir in beiden Gruppen bei ungefähr der Hälfte der Patienten fest.

Bei den PV war die Anzahl der Tierbissverletzungen etwas höher als bei den SV, dieser Unterschied war jedoch nicht signifikant. Der Anteil der Menschenbissverletzungen war vergleichbar. Die restlichen Patienten beider Gruppe erlitten Schnitt-, respektive Stichverletzungen, Fremdkörperinokulationen und andere Bagatelltraumata oder konnten sich an kein auslösendes Ereignis erinnern.

In beiden Gruppen gab es Infektionen unterschiedlicher Ausprägung an verschiedenen Lokalisationen der Hand. In der PVGruppe waren in 13 Fällen die Finger und in 3 Fällen die Hand 
betroffen. Die Infektionen an den Fingern unterteilten sich in 7 phlegmonöse Weichteilinfektionen, 3 nicht näher spezifizierte Panaritien, 2 Osteomyelitiden und ein Gelenkempyem. Am Handrücken bestand bei 2 Patienten eine Phlegmone und bei einem Patienten ein lokale Entzündung.

In der SV-Gruppe waren die Finger in 9 Fällen und die Hand in 3 Fällen betroffen. An den Fingern lagen 7 phlegmonöse Weichteilinfektionen, eine Osteomyelitis und ein Gelenkempyem vor. An der Hand zeigte sich bei 2 Patienten eine Phlegmone am Handrücken und bei einem Patienten in der Hohlhand.

In der PV-Gruppe waren 7 Patienten vor der Aufnahme in unsere Klinik mit Antibiotika vorbehandelt worden, in der SV-Gruppe traf dies auf 4 Patienten zu. Die restliche Patienten erhielten die erste Antibiotikagabe durch uns. Die mittlere Vorbehandlungsdauer betrug in der PV-Gruppe 4,4 Tage (3-14 Tage), in der SVGruppe entsprechend 2,5 Tage (1-7 Tage).

Die Latenz zwischen den infektionsauslösenden Ursachen und der Operation lag in der PV-Gruppe im Durchschnitt bei 6,3 Tagen (0-23 Tage) und in der SV-Gruppe bei 4,8 Tagen (1-7 Tage). Bei der statistischen Auswertung der Daten zur Dauer des stationären Aufenthalts, des Durchschnittsalters und der Gesamtzufriedenheit der beiden Gruppen kam der doppelte t-test zur Anwendung, bei allen restlichen Berechnungen haben wir den exakten Test nach Fisher verwendet.

\section{Ergebnisse}

$\nabla$

Keiner der in die Studie eingeschlossenen Patienten musste nach dem PV, respektive SV noch einmal nachoperiert werden (๑ Tab. 2).

9 Patienten mit PV und 4 Patienten mit SV waren uneingeschränkt zufrieden mit ihrer Narbe und gaben die Bewertung „gut“ ab. Die restlichen Patienten bewerteten ihre Narbe als „schlecht“ ( $\bullet$ Tab. 2). Der führende Grund hierfür war in beiden Gruppen eine „Kälteempfindlichkeit“ der Narbe gefolgt von der Begründung „Taubheit“ der Narbe. Die Beweglichkeit der operierten Hand war für beide Gruppen vergleichbar, 11 der PV-Patienten und 9 der SV-Patienten werteten diese als genauso gut wie vor der Operation ( $\bullet$ Tab. 2).

Die Dauer des Krankenhausaufenthaltes war im Durchschnitt für die Patienten mit PV mit 3,0 Nächten (0-7) signifikant kürzer als für die Patienten mit SV (5,1 Nächte, 2-7) ( $p=0,048)$ ( $\bullet$ Abb. 1). Sowohl die PV- als auch die SV-Patienten gaben ihrer Behandlung im Durchschnitt die Note 2,1 (1-5) ( $\bullet$ Abb. 2).

\section{Diskussion \\ $\nabla$}

Unsere Studie zeigt, dass nach einem adäquaten Débridement einer Infektion an der Hand ein primärer Wundverschluss ohne Nachteile für den Patienten möglich ist. Dadurch kann der stationäre Aufenthalt des Patienten signifikant verkürzt werden. Unbedingte Voraussetzung für dieses Vorgehen ist ein adäquates, radikales Débridement des infizierten Gewebes und eine ausgiebige Spülung der Wunde. Den Stellenwert eines Débridements unterstreichen erste Hinweise, dass nach einem entsprechenden Débridement von infiziertem Gewebe auf eine antibiotische Therapie bei Handinfektionen verzichtet werden kann [9]. Traditionsgemäß werden infizierte Wunden nicht primär vernäht und im Rahmen einer Zweit-Operation sekundär verschlossen [10]. Dieses Vorgehen wird mit einer erleichterten Drainage
Tab. 2 Ergebnisse der Telefonbefragung hinsichtlich Reoperationen, Zufriedenheit mit der Narbe und Beweglichkeit der operierten Hand.

\begin{tabular}{|c|c|c|c|}
\hline & $\begin{array}{l}\text { Primärver- } \\
\text { schluss }\end{array}$ & $\begin{array}{l}\text { Sekundär- } \\
\text { verschluss }\end{array}$ & p-Wert \\
\hline Reoperationen & $0 / 16$ & $0 / 12$ & 1,00 \\
\hline $\begin{array}{l}\text { uneingeschränkte Zufrieden- } \\
\text { heit mit der Narbe }\end{array}$ & $9 / 16$ & $4 / 12$ & 0,28 \\
\hline $\begin{array}{l}\text { vollständiges Wiedererlangen } \\
\text { der Beweglichkeit der Hand }\end{array}$ & $11 / 16$ & $9 / 12$ & 1,00 \\
\hline
\end{tabular}

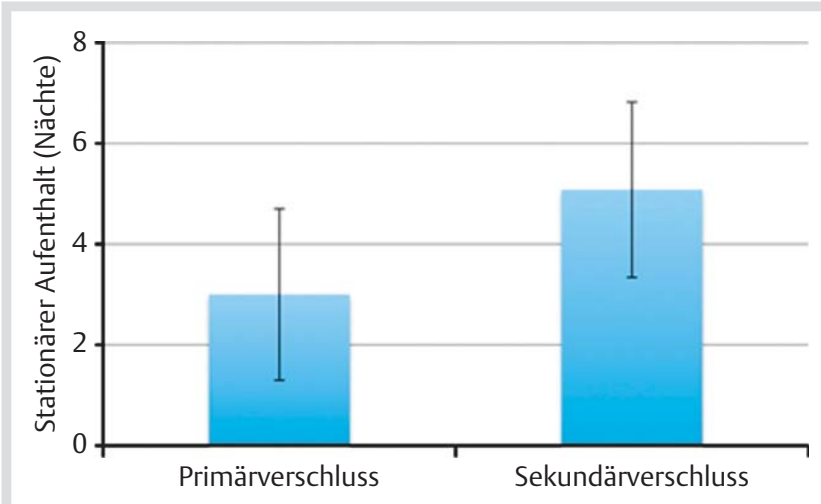

Abb. 1 Durchschnittliche Dauer des stationären Aufenthalts in Nächten für Patienten mit Primärverschluss $(3,0)$ und Patienten mit Sekundärverschluss $(5,1)$. Mean \pm SEM, $p=0,048$.

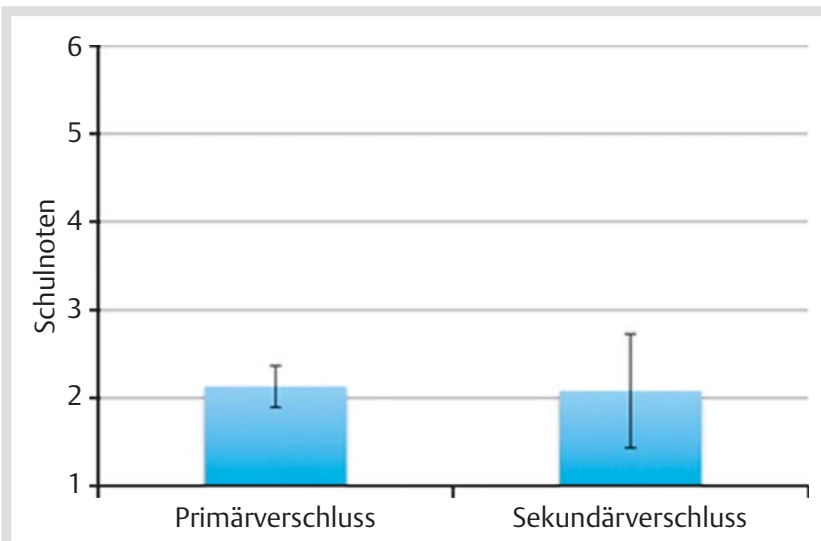

Abb. 2 Zufriedenheit der beiden Patientengruppen. Sowohl die PV- als auch die SV-Patienten gaben ihrer Behandlung im Durchschnitt die Note 2,1. Mean \pm SEM.

von Wundsekret und einem geringeren Risiko einer Reinfektion begründet [11]. Demgegenüber steht die Überzeugung, dass ein Primärverschluss einer infizierten Wunde möglich ist, sofern ein radikales und vollständiges Débridement des infizierten Gewebes vorgenommen wurde.

De Melker et al. untersuchten im Rahmen einer Meta-Analyse Hundbissverletzungen hinsichtlich Risikofaktoren, der Inzidenz von Infektionen, dem Nutzen einer prophylaktischen Antibiotikabehandlung und der Sicherheit von Primärverschlüssen [12]. Es stellte sich heraus, dass eine Hundebissverletzung nach adäquatem Débridement ohne erhöhtes Infektionsrisiko primär verschlossen werden kann. Maimaris et al. führten eine prospektive Studie durch, bei der Hundebissverletzungen nach einem chirurgischen Débridement und einer Spülung der Wunde entweder primär verschlossen oder offen gelassen wurden [13]. 
Die Hand war dabei signifikant häufiger betroffen als andere Körperregionen. Auch hier zeigte sich kein Unterschied in der Infektionsrate, diese lag in der Gesamtpopulation bei 7,7\%. In den Studien von de Melker und Maimaris handelte es sich jedoch nicht um manifeste Infektionen wie in unserer Studie, sondern um frische Verletzungen. Singer et al. bestätigten diese Tendenz in einer Meta-Analyse von 7 Studien, die sich mit dem Primärverschluss kutaner Abszesse befassten. Für die Auswertung wurden Abszesse unabhängig ihrer Lokalisation am Körper untersucht, wobei der Großteil in der Anogenitalregion lag [14]. Die Autoren zeigten, dass die Heilungsdauer nach Primärverschluss signifikant kürzer war als nach einem Sekundärverschluss. Zudem konnte keine erhöhte Reinfektionsrate nach Primärverschluss festgestellt werden.

Bongartz et al. führten bereits im Jahre 1988 eine prospektive Studie durch, bei der kontaminierte Wunden durch eine Primärnaht verschlossen wurden [15]. Nur bei 2 von 48 Patienten kam es zu einer Infektion der Wunde, die nach dem Öffnen der Nähte problemlos abheilte. Die Autoren kamen zu dem Ergebnis, dass ein Primärverschluss von kontaminierten Wunden ein nur geringes Risiko für Infektionen birgt und, verglichen mit Sekundärverschlüssen, zudem kostengünstiger für das Gesundheitssystem und angenehmer für den Patienten ist. Auch in dieser Studie handelte es sich nicht um manifeste Infektionen, sondern ausschließlich um kontaminierte Wunden. Unsere Studie ist die erste Arbeit, die sich mit Primärverschlüssen bei manifesten Infektionen an der Hand beschäftigt. Neben den genannten positiven Effekten hatte eine Primärnaht in der Studie von Bongartz et al. den Vorteil eines im Allgemeinen ästhetisch besseren Ergebnisses. Ein eventuell schlechteres ästhetisches Ergebnis bei SV könnte dadurch entstehen, dass bei einem „second-look“ häufig nochmals Gewebe débridiert wird und somit die Spannung der Wunde tendenziell zunimmt.

Auch in unserer Studie werteten, verglichen mit der SV-Gruppe, tendenziell mehr Patienten der PV-Gruppe ihre Narbe als „gut“, dieser Unterschied war jedoch nicht signifikant. Unseren Patienten erschien weniger die Ästhetik der Narbe, als vielmehr die Funktionalität (keine Kontraktur, keine Taubheit, keine Kälteempfindlichkeit) als wichtig.

Diese Qualitätseinschätzung der Narbe durch die Patienten ist sicherlich sehr subjektiv und hängt eher von den persönlichen Ansprüchen als von objektiven Kriterien ab.

Bei allen Patienten mit einer Handinfektion ist eine tägliche Nachbeobachtung der Wunde notwendig. Alle Patienten, die in diese Studie aufgenommen wurden, wurden bis zum Abklingen der labortechnischen und klinischen Infektionszeichen täglich gesehen, ungeachtet, ob die Patienten ambulant oder stationär geführt wurden.

Unsere Studie zeigt, dass PV-Patienten im Durchschnitt signifikant kürzere stationäre Aufenthalte hatten als SV-Patienten. Dies lässt sich mit einer notwendigen zweiten Operation bei Sekundärverschlüssen, die normalerweise ca. 2-3 Tage nach der initialen Operation durchgeführt wird, erklären. Primäres Ziel ist die optimale Versorgung der Patienten mit einer Minimierung der physischen und psychischen Belastung durch die Behandlung. Ein verantwortungsvoller Umgang mit den Ressourcen Operationszeit und Liegedauer ist jedoch sicher wünschenswert. Alleine die Kosten für das Material der temporären Defektdeckung und der Zweitoperation bewegen sich im 3-stelligen Bereich.

In unserer Studie war die Patientenzufriedenheit der beiden Behandlungsgruppen annähernd gleich gut. Dies lässt vermuten, dass die Zufriedenheit der Patienten eher mit dem Ergebnis der Operation und eventuell auch mit der Betreuung des Patienten durch das medizinische Personal und nicht mit der Länge des Krankenhausaufenthalts zusammenhängt.

Genauso verhält es sich bei dem Wiedererlangen der präoperativen Beweglichkeit der Hand, ein Parameter, der subjektiv vom Patienten abgefragt wurde und bei dem es keine signifikanten Unterschiede zwischen den beiden Gruppen gab.

Bei Handinfektionen ist nach einem radikalen Débridement ein Primärverschluss der Wunde ohne Nachteile für den Patienten möglich. Sollte bei ausgedehnten Handinfektionen ein radikales Débridement z.B. aufgrund von betroffenen funktionellen Strukturen nicht möglich sein, kann im Zweifelsfall ein „secondlook" in Betracht gezogen und geplant werden. In diesem Fall kann jedoch - unter Einplanung eines „second-looks“ - ebenfalls ein adaptierender Primärverschluss durchgeführt werden. Dies hat den Vorteil einer Defektdeckung durch körpereigenes Material und die nicht zwingend erforderliche Zweitoperation, falls die Infektion abklingt.

Unsere Studie ist unter verschiedenen Gesichtspunkten limitiert. Erstens beinhaltete das Follow-up keine klinische Nachuntersuchung. Alle Patienten werden von uns regelmäßig zur Nachsorge einbestellt. Das Ausmaß der Infektion und die Art der Verletzung innerhalb der Gruppen waren jedoch zu heterogen, als dass ein weiterführender klinischer Vergleich sinnvoll gewesen wäre. Zweitens hat die Studie ein retrospektives Design, die Behandlung und somit die Zuteilung zu den beiden Gruppen war jedoch durch die Präferenz des diensthabenden Operateurs festgelegt und erfolgte unabhängig von der Vorgeschichte und dem Ausmaß der Infektion. Drittens beinhaltet diese Studie nur eine kleine Fallzahl. Unsere Klinik ist ein Krankenhaus der Maximalversorgung in einer dicht besiedelten Region. Daher sind wir häufig nicht die primäre Anlaufstelle für Patienten mit Handinfektionen, sondern bekommen vermehrt Patienten vorgestellt, die bereits voroperiert sind.

Unserer Ansicht nach wäre das Patientengut durch den Einschluss der voroperierten Patienten zu heterogen geworden.

\section{Schlussfolgerung}

\section{$\nabla$}

Unsere Studie zeigt, dass nach einem radikalen, vollständigen Débridement einer Handinfektion die Wunde ohne erhöhtes Risiko für den Patienten primär verschlossen werden kann. Dadurch wird die Länge des Krankenhausaufenthalts bei diesem Patientengut signifikant verkürzt, verglichen mit Patienten mit Sekundärverschluss, was sowohl ein Vorteil für den betroffenen Patienten darstellt, als auch aus gesundheits-ökonomischer Sicht erstrebenswert scheint.

Primär- und Sekundärverschlüsse bei Handinfektionen zeigen beide gute klinische Ergebnisse und führen zu einer hohen Patientenzufriedenheit.

Es ist zu mutmaßen, dass in manchen Fällen aufgrund von Unerfahrenheit im Umgang mit Handinfektionen und der damit verbundenen Angst vor einem inadäquaten Débridement zu einem Sekundärverschluss tendiert wird. Aus diesem Grund ist es unserer Meinung nach zu fordern, dass die Behandlung von Handinfektionen in die Hände von Experten gegeben wird.

Interessenkonflikt: Nein 


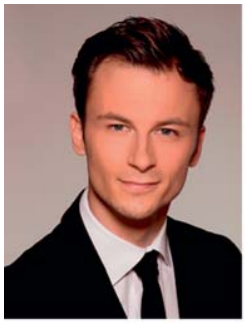

Dr. med. Daniel Christopher Schmauß Geb. am 29.06.1982 in Augsburg. Studium der Humanmedizin an der Ludwig-Maximilians-Universität München von 2002 bis 2009 mit Auslandsaufenthalten in New York und Chicago. Stipendiat der Harvard-Munich Medical Alliance. 2008-2009 Ablegen der 3 amerikanischen Staatsexamina (USMLE). 2009 Promotion an der Klinik und Poliklinik für Kardiologie der LMU München (Prof. Dr. med. G. Steinbeck). 2009-2011 Assistenzarzt an der Klinik und Poliklinik für Herzchirurgie der LMU München (Prof. Dr. med. B. Reichart). 2011-2012 Notaufnahmerotation an der Klinik für Unfall-, Wiederherstellungs- und Handchirurgie, Zentralklinikum Augsburg (Prof. Dr. med. Dr. h.c. E. Mayr). Seit Mai 2011 Assistenzarzt an der Klinik und Poliklinik für Plastische Chirurgie und Handchirurgie am Klinikum rechts der Isar München (Prof. Dr. med. H-G Machens).

\section{Literatur}

1 Leung NL, Lee SK. Viral infections of the hand. Current Orthopaedic Practice 2010; 21: 568-572

2 McDonald LS, Bavaro MF, Hofmeister EP et al. Hand infections. J Hand Surg Am 2011; 36: 1403-1412

3 Reh-Plass S, Schaller E. Stellenwert der Frühintervention bei Handinfektionen. Handchir Mikrochir Plast Chir. 1991; 23: 214-217
4 Reichert B, Oeynhausen-Petsch P, Mailänder P. Die Handinfektion als Folge einer Bagatellisierung von Minimalverletzungen. Handchir Mikrochir Plast Chir. 2007; 39: 124-127

5 Reichert B, Zöphel O, Möller M et al. Behandlungskosten durch unterschätzte Handinfektionen. Handchir Mikrochir Plast Chir. 2001; 33: 354-358

6 Rigopoulos N, Dailiana ZH, Varitimidis S et al. Closed-space hand infections: diagnostic and treatment considerations. Orthop Rev (Pavia) 2012; 4: e19

7 Singer AJ, Taira BR, Chale $S$ et al. Primary versus secondary closure of cutaneous abscesses in the emergency department: a randomized controlled trial. Acad Emerg Med 2013; 20: 27-32

8 Ellis $M$. Incision and primary suture of abscesses of the anal region. Proc R Soc Med 1960; 53: 652-654

9 Manoli T, Rahmanian-Schwarz A, Konheiser $K$ et al. The role of antibiotics after surgical treatment of simple hand infections: a prospective pilot study. J Invest Surg 2013; 26: 229-234

10 Kall S, Vogt PM. Chirurgische Therapie von Infektionen der Hand - Teil I. Chirurg 2005; 76: 615-628

11 Macfie J, Harvey J. The treatment of acute superficial abscesses: a prospective clinical trial. Br J Surg 1977; 64: 264-266

12 De Melker HE, de Melker RA. Dog bites: publications on risk factors, infections, antibiotics and primary wound closure. Ned Tijdschr Geneeskd 1996; 140: 709-713

13 Maimaris C, Quinton DN. Dog bite lacerations: a controlled trial of primary wound closure. Arch Emerg Med 1988; 5: 156-161

14 Singer AJ, Thode HC, Chale S et al. Primary closure of cutaneous abscesses: a systematic review. American Journal of Emergency Medicine 2011; 29: 361-366

15 Bongartz W, Lindner HO, Schumpelick V. Primärnaht von älteren und kontaminierten Wunden. Eine prospektive klinische Studie. Chirurg. 1988; 59: 767-770 